\title{
Atividade de promoção à saúde em um grupo de idosos
}

Health promotion activity in an elderly people group

\author{
Maciela Ferreira dos Santos ${ }^{1}$ \\ Regiane Kelly Lopes Silva ${ }^{2}$ \\ José Henrique Rocha da Costa ${ }^{3}$ \\ Jeisabelly Adrianne Lima Teixeira ${ }^{4}$ \\ Ernandes Gonçalves Dias ${ }^{5}$
}

\section{RESUMO}

Atividades de promoção à saúde consistem em meios de buscar a melhoria da qualidade de vida de uma população. O objetivo deste texto é descrever a experiência de uma atividade realizada com um grupo de idosos, desenvolvida por acadêmicos dos cursos de bacharelado e licenciatura em Educação Física de uma faculdade particular. As atividades aconteceram em um distrito, zona rural da cidade de Porteirinha, Minas Gerais, em 2017, no Centro de Referência em Assistência Social. Foram desenvolvidas atividades físicas (alongamento dos membros superiores e inferiores, exercícios de trabalho com a coordenação fina, flexibilidade, entre outros), recreativas (músicas) e oficina de educação em saúde com abordagem do tema "Importância da atividade física para promoção do envelhecimento ativo e saudável". A realização de atividades recreativas e educação em saúde em grupo para os idosos facilitou a socialização do conhecimento e das experiências de cada um, além da oportunidade de promover interação social entre os idosos. Ações nesse sentido devem ser incentivadas e valorizadas, pois ajudam os idosos a manterem sua autonomia.

Palavras-chave: Promoção da saúde. Educação em saúde. Saúde do idoso. Educação Física.

\begin{abstract}
Health promotion activities consist of means to seek to improve the quality of life of a population. This text aims to describe the experience of an activity performed with a group of elderly people, developed by academics of the bachelor and Physical Education degree courses of a particular university. The activities were conducted from the perspective of popular health education and took place in a district, rural area of the city of Porteirinha, State of Minas Gerais, Brazil, in 2017, at the Reference Center for Social Assistance. Physical activities (stretching of the upper and lower limbs, exercises for fine coordination, flexibility, among others), recreational activities (music) and a health education workshop with the theme "Importance of physical activity for the promotion of active aging it's healthy". The

1 Graduanda em Educação Física no Instituto Superior de Educação Verde Norte, Minas Gerais, Brasil (santosmaciela@gmail.com).

2 Graduanda em Educação Física no Instituto Superior de Educação Verde Norte, Minas Gerais, Brasil (regianekelly25@gmail.com).

3 Graduando em Educação Física no Instituto Superior de Educação Verde Norte, Minas Gerais, Brasil (henrique50040@hotmail.com).

4 Mestranda em Ciência da Educação na Universidad Tecnológica Intercontinental, Paraguai; professora no curso de Graduação em Enfermagem na Faculdade Verde Norte, Minas Gerais, Brasil (jeisabellyadrianne@gmail.com).

${ }^{5}$ Mestre em Ciências pela Universidade de São Paulo, Brasil; professor no curso de Graduação em Enfermagem da Faculdade Verde Norte, Minas Gerais, Brasil; coordenador de Atenção Básica no município de Monte Azul, Minas Gerais, Brasil (ernandesgdias@yahoo.com.br).
\end{abstract}


realization of recreational activities and group health education for the elderly facilitates the socialization of the knowledge and experiences of each one, besides the opportunity to promote social interaction among the elderly. Actions in this sense should be encouraged and valued, as they help the elderly to maintain their autonomy.

Keywords: Health promotion. Health education. Health of the elderly. Physical Education.

\section{INTRODUÇÃO}

O envelhecimento humano é um fenômeno natural, social, irreversível e mundial, que não pode ser caracterizado apenas pela degeneração biológica, pois a partir dele há problemas e limitações de ordem econômica, condições políticas, históricas e socioculturais que singularizam o processo de envelhecer (SANTOS; FORONI; CHAVES, 2009; MELO et al., 2009).

O período entre 1970 e 2025 é considerado a era do envelhecimento. No Brasil, é considerado idoso todo indivíduo que possui 60 anos ou mais de idade (BRASIL, 2003; GÓIS; VERAS, 2010). Atualmente $13 \%$ da população brasileira têm mais de 60 anos e as estimativas apontam que até 2060 o número de idosos no Brasil chegue a 34\% (IBGE, 2014).

Com o aumento do número de idosos, existe a necessidade da adequação e preparo dos serviços de saúde, visando a sua estruturação, formação e qualificação profissional para o atendimento dessa nova demanda (SIQUEIRA et al., 2009), uma vez que a população idosa é grande usuária de serviços de saúde (SALIN et al., 2011; PILGER; MENON; MATHIAS, 2013).

A prática de atividade física regular na perspectiva do envelhecimento saudável e ativo se constitui como uma condição favorável na manutenção da saúde e na prevenção de Doenças Crônicas não Transmissíveis (DCNT) (MALTA; SILVA JÚNIOR, 2013), tendo em vista os benefícios físicos, psicológicos e sociais (CAMBOIM et al., 2017).

Durante o processo de envelhecimento ocorrem muitas mudanças no organismo humano, elas podem ser agravadas dependendo do estilo de vida de cada pessoa. $\mathrm{O}$ estilo de vida adotado pelo indivíduo também pode resultar em novos problemas para a saúde, contribuindo inclusive para uma maior incidência de doenças neurodegenerativas como o mal de Parkinson e Alzheimer (MARI et al., 2016; FALCO et al., 2016).

Nesse sentido, um grupo de acadêmicos da Faculdade Verde Norte (Favenorte) do curso de licenciatura e bacharelado em Educação Física realizaram uma atividade de educação em saúde com um grupo de idosos em Porteirinha, Minas Gerais. A atividade visou proporcionar 
atividades de lazer no Centro de Referência em Assistência Social (CRAS) no intuito de observar os benefícios físicos decorrentes da prática do exercício físico entre os idosos do grupo "Viver a Vida".

Essa ação pode ser vista, inclusive, como uma atividade de extensão (um dos três pilares do ensino superior) com intuito de aproximar a Instituição de Ensino Superior (IES) com a comunidade rural adjacente à Faculdade. Segundo Dias et al. (2016), a extensão universitária é um pilar que deve nortear permanentemente o ensino superior, visto que, por meio dela, a IES consegue formar profissionais envolvidos com as questões de interesse social. Dessa forma o objetivo deste trabalho foi descrever a experiência de uma atividade realizada com um grupo de idosos, desenvolvida por acadêmicos dos cursos de bacharelado e licenciatura em Educação Física de uma Faculdade particular.

\section{Relato da experiência}

Este estudo trata-se de um relato de natureza descritiva, que aborda a experiência de um grupo de acadêmicos do Curso de Graduação (bacharelado e licenciatura) em Educação Física da Favenorte junto a um grupo de idosos moradores da zona rural de Porteirinha, Minas Gerais. As atividades foram realizadas em 19 de outubro de 2017, no período vespertino, e teve duração aproximada de três horas. Na ocasião, reuniram-se no CRAS 20 idosos, sendo 4 do sexo masculino e 16 do sexo feminino.

Esse grupo se reúne quinzenalmente no CRAS, onde são realizadas atividades como palestras referentes à alimentação e bem-estar, aulas de dança, sorteio de brindes e gincanas, entre outras atividades de recreação e lazer, por profissionais do CRAS. Dias et al. (2017) entendem que a criação de grupos de convivência de idosos para atividades pode ser uma estratégia para motivá-los para o autocuidado.

Para efetivar a ação, num primeiro momento foi estabelecido contato com o responsável pelo CRAS, que assinou autorização para realização do encontro. O CRAS é um ambiente de fácil acesso a todos no distrito, tem amplo espaço físico e acessibilidade para pessoas com deficiência física ou mobilidade reduzida.

A ação ocorreu em uma sala ampla, arejada e iluminada com a disposição de cadeiras em círculo para facilitar a interação e a realização das atividades propostas. 
As atividades planejadas tiveram como objetivo favorecer a socialização entre os idosos e englobaram quatro etapas. A etapa inicial envolveu atividades físicas (alongamento dos membros superiores, inferiores e lombar) em posição sentada, com ajuda dos interventores com duração de 30 minutos. Para alongamento dos membros inferiores, foi realizado exercício de alongamento da panturrilha esticada com o auxílio de uma cadeira; para os membros superiores, foram realizados exercícios como antebraço esticado, flexão lateral de pescoço, rotação de pescoço, antebraço esticado, ombro lateral esticado; e na região lombar aplicou-se alongamento de coluna. Vale ressaltar que se buscou trabalhar os grupos musculares de forma coordenada.

Na etapa secundária, foi realizada uma dança com movimentos de baixa intensidade com o objetivo de trabalhar a coordenação motora e noção de espaço e equilíbrio. Nessa etapa, trabalhou-se com a música Happy, de Pharrel Williams, por considerar-se adequada para a realização de movimentos variados. Nesse momento, os participantes fizeram uma sequência de movimentos orientados pelos acadêmicos, com duração de 60 minutos com curtos intervalos.

No momento das atividades foi possível verificar interesse, participação efetiva e grande entusiasmo na execução dos movimentos de alongamento e da dança que, certamente, influenciaram no desenvolvimento do senso reflexivo e participativo dos idosos.

A terceira etapa se tratou de uma oficina de educação em saúde com abordagem do tema “Importância da atividade física para promoção do envelhecimento ativo e saudável". Nessa etapa, os idosos se posicionaram assentados, fizeram relatos de suas vidas e realizaram avaliação da experiência que vivenciaram. Essa fase teve duração de 60 minutos. Nessa etapa foi possível verificar a diversidade cultural, as experiências de vida, os anseios e desejos dos idosos. Foi um momento propício para troca de experiência e observação quanto ao prazer deles em estar no grupo e realizar as atividades propostas. Isso permitiu relações interpessoais proveitosas, tanto para os acadêmicos quanto para os próprios idosos. Esse momento favoreceu o diálogo e a discussão entre todos os participantes.

A quarta etapa se tratou da avaliação (conversa com participantes para expor suas impressões) e encerramento da ação com oferecimento de um lanche para confraternização e interação. Foram servidos sucos naturais, frutas e bolo, oferecido pelo CRAS. A avaliação se deu baseada no princípio do diálogo, menos formal, entre os participantes e os acadêmicos. 
As ações realizadas foram organizadas a partir da percepção de que a prática de exercícios físicos pelas pessoas idosas melhora a capacidade funcional, o equilíbrio, a força, a coordenação e a velocidade de movimento, e contribui para segurança e prevenção de quedas entre os idosos.

Para os acadêmicos participantes a ação foi significativa na perspectiva de ser uma possibilidade de desenvolvimento de papéis e promoção de um ambiente de formação centrado na articulação entre o ensino e a responsabilidade social do graduando, que também se pretende estimular para a vida profissional, sem desconsiderar a importância do desenvolvimento da extensão também para a Instituição de Ensino.

\section{DISCUSSÃO}

Evidências científicas indicam que a participação de idosos em programas de atividades físicas é uma forma independente para reduzir e/ou prevenir uma série de declínios funcionais associados ao envelhecimento (NELSON et al., 2007; CAMPOS et al., 2014; FONSECA et al., 2016).

Os benefícios de um comportamento ativo do idoso são perceptíveis nas esferas biológica, psicológica e social, com destaque para aumento/manutenção da capacidade aeróbia, aumento/manutenção da massa muscular, redução da mortalidade, prevenção de doenças coronarianas, melhora do perfil lipídico, redução da massa gorda e risco de sarcopenia, prevenção/controle da diabete tipo II e Hipertensão Arterial Sistêmica (HAS); redução da ocorrência de Acidente Vascular Endocefálico (AVE) (MACIEL, 2010).

A atividade física desempenha um papel importante na vida do idoso, previne doenças, garante bem-estar e longevidade. De maneira geral melhora na resistência, flexibilidade, agilidade, coordenação motora e equilíbrio com impacto positivo no sistema imunológico e na prevenção de doenças como diabetes, artrite, doenças cardíacas, além de auxiliar no tratamento de condições psíquicas como a depressão. Sendo assim, deve-se estimular a população idosa a praticar atividade física para promover a melhoria de seu estado de saúde (SANTOS, 2014).

A atividade física é também prevenção primária do câncer de mama e cólon, reduz a ocorrência de demência, melhora a autoestima e a autoconfiança, diminui a ansiedade e o estresse; melhora o estado de humor e a qualidade de vida (MACIEL, 2010; INCA, 2018). Sua prática proporciona benefícios também psicológicos. Um estudo realizado com 150 
idosos de ambos os sexos que praticavam exercícios físicos em duas universidades públicas do sul do Brasil demonstrou que a participação nessas atividades provocou percepção positiva em relação à autoimagem e autoestima dos frequentadores (MEURER; BENEDETTI; MAZO, 2009).

No estudo realizado por Yokoyama, Carvalho e Vizzotto, em 2006, com 10 homens e 20 mulheres com idades entre 60 e 82 anos, frequentadores de um centro de referência do $\mathrm{ABC}$ Paulista, o estado emocional positivo apareceu em 29,3\% das respostas dos participantes. Esse percentual foi maior entre as mulheres, que demonstraram maior preocupação com o bem-estar.

À prática de atividade física regular atribui-se, também, a redução do risco de morte prematura, câncer de cólon e mama e diabetes tipo II. Além disso, atua na prevenção e controle da HAS e do ganho de peso ponderal, auxilia na prevenção ou controle da osteoporose, promovendo bem-estar, reduz o estresse, a ansiedade e estados depressivos (FERREIRA, 2017).

A experiência vivenciada com o grupo de idosos possibilitou reflexões pertinentes quanto à existência e execução de programas assistenciais e grupos de convivência a essa faixa etária. É importante a realização de um trabalho multiprofissional de educação em saúde de idosos para que, assim, seja oportunizado e estimulado o desenvolvimento de suas habilidades cognitivas, físicas, emocionais e sociais.

Nesse sentido, a educação em saúde é uma importante ferramenta para promoção do envelhecimento ativo, visto que é possível prevenir e controlar doenças, proporcionar atividade cognitiva, participação social e comportamentos de saúde. Assim, cabe a ela promover hábitos de vida saudáveis ao permitir a troca de conhecimentos e saberes e mobilizar recursos individuais e coletivos (MALLMANN et al., 2015).

Desse modo, é relevante destacar a importância das práticas de educação em saúde quanto ao estímulo provocado no idoso a buscar independência, autonomia e satisfação com a vida, como também ser um espaço de convívio social, onde se utiliza o diálogo para a promoção da saúde.

Diante da ação realizada, reforça-se ser importante atentar para uma formação acadêmica que permita uma visão integral dos sujeitos e uma formação profissional mais humanizada, que abarque uma visão holística e integral da realidade social, no sentido de que os estudantes 
possam ser estimulados a participarem de atividades extensionistas (OLIVEIRA; ALMEIDA JÚNIOR, 2015).

\section{CONSIDERAÇÕES FINAIS}

A realização de atividades recreativas e de educação em saúde em grupo para os idosos facilita a socialização do conhecimento e das experiências de cada um, além de representar uma oportunidade para promover interação entre eles. Observou-se sensação de prazer e a alegria entre os idosos participantes da ação.

O fato de a ação ser realizada em um povoado pequeno, com poucos recursos, pode ser um limitador, porém se mostrou positiva, visto a falta de atividades diferenciadas para promoção do envelhecimento saudável. Ações nesse sentido devem ser incentivadas com o intuito de que o envelhecimento ativo e saudável não seja privilégio de idosos que vivem em regiões de melhor desenvolvimento econômico ou inseridos em regiões de acesso físico facilitado.

Essas experiências devem ser incentivadas e valorizadas, pois ajudam os idosos a manterem sua autonomia. Além da importância da representação social que essas ações têm em mantêlos convivendo em grupo, elas têm auxiliado a melhorar sua qualidade de vida, conferindo maior resistência e prevenção às quedas e fraturas nessa população.

Numa outra perspectiva, é sempre importante a relação que se estabelece entre os sujeitos e acadêmicos em função das ações de extensão, que possibilitam a inserção de estudantes de graduação na comunidade e nos espaços sociais em que estão inseridas as pessoas para potencializar a aprendizagem e atender as necessidades ali existentes, considerando, é claro o perfil do profissional em formação.

\section{REFERÊNCIAS}

BRASIL. Presidência da República. Casa Civil. Lei no 10.741 , de $1^{\circ}$ de outubro de 2003. Dispõe sobre o Estatuto do Idoso e dá outras providências. Diário Oficial da União, Brasília, DF. 3 out. 2003.

CAMBOIM, F. E. F. et al. Benefícios da atividade física na terceira idade para a qualidade de vida. UFPE on line: Revista de Enfermagem, Recife, v. 11, n. 6, p. 2.415-2.422, jun. 2017. Doi: 10.5205/reuol.10827-96111-1-ED.1106201721. 
CAMPOS, A. C. V. et al. Qualidade de vida de idosos praticantes de atividade física no contexto da estratégia saúde da família. Texto Contexto Enferm., Florianópolis, v. 23, n. 4, out./dez., 2014. Doi: 10.1590/0104-07072014002060013.

DIAS, E. G. et al. Desafios da prática do autocuidado do idoso portador de diabetes mellitus tipo 2. Sustinere, Rio de Janeiro, v. 5, n. 1, p. 38-53, jan./jun. 2017. Doi: https://doi.org/10.12957/sustinere.2017.26483.

DIAS, E. G. et al. Implantação de uma comissão de extensão em uma instituição de ensino superior privada. Revista Saúde e Desenvolvimento, v. 9, n. 5, p. 20-36, jan./jun. 2016. https://www.uninter.com/revistasaude/index.php/saudeDesenvolvimento/article/view/540/306

FALCO, A. et al. Doença de Alzheimer: hipóteses etiológicas e perspectivas de tratamento. Química Nova, São Paulo, v. 39, n. 1, jan. 2016. Doi: 10.5935/0100-4042.20150152.

FERREIRA, G. B. Atividade física e envelhecimento: benefícios dos exercícios proprioceptivos na prevenção de quedas em pessoas idosas. 2017. 29 f. Trabalho de Conclusão de Curso (Graduação em Educação Física) - União Metropolitana para Educação e Cultura, Salvador, 2017.

FONSECA, S. C. et al. O envelhecimento ativo e seus fundamentos. São Paulo: Portal Edições, 2016.

GÓIS, A. L. B.; VERAS, R. P. Informações sobre a morbidade hospitalar em idosos nas internações do Sistema Único de Saúde do Brasil. Ciênc. Saúde Coletiva, Rio de Janeiro, v. 15, n. 6, p. 2.859-2.869, 2010. Doi: 10.1590/S1413-81232010000600023.

IBGE. Instituto Brasileiro de Geografia e Estatística. Projeções da população do Brasil. 2014. Disponível em: https://www.ibge.gov.br/apps/populacao/projecao/. Acesso em: 15 jun. 2019.

INCA. Instituto Nacional do Câncer. Atividade física. 2018. Disponível em: https://www.inca.gov.br/causas-e-prevencao/prevencao-e-fatores-de-risco/atividade-fisica.

Acesso em: 15 jun. 2019.

MACIEL, M. G. Atividade física e funcionalidade do idoso. Motriz, Rio Claro, v. 16, n. 4, p.1024-1032, out./dez., $2010 . \quad$ Disponível em: http://www.scielo.br/pdf/motriz/v16n4/a23v16n4.pdf. Acesso em: 15 maio 2019.

MALLMANN, D. G. et al. Educação em saúde como principal alternativa para promover a saúde do idoso. Ciênc. Saúde Coletiva, Rio de Janeiro, v. 20, n. 6, p. 763-1772, 2015. Doi: 10.1590/1413-81232015206.0238201.

MALTA, D. C.; SILVA JÚNIOR, J. B. O plano de ações estratégicas para o enfrentamento das doenças crônicas não transmissíveis no Brasil e a definição das metas globais para o enfrentamento dessas doenças até 2025: uma revisão. Epidemiol. Serv. Saúde, Brasília, v. 22, n. 1, p. 151-164, 2013. Doi: 10.5123/S1679-49742013000100016.

MARI, F. R. et al. O processo de envelhecimento e a saúde: o que pensam as pessoas de meia-idade sobre o tema. Rev. Bras. Geriatr. Gerontol., Rio de Janeiro, v. 19, n. 1, p. 35-44, 2016. Doi: 10.1590/1809-9823.2016.14122. 
MELO, M. C. et al. A educação em saúde como agente promotor de saúde. Ciênc. Saúde Coletiva, Rio de Janeiro, v. 14, supl. 1, p. 1.579-1.586, 2009. Doi: 10.1590/S141381232009000800031 .

MEURER, S. T.; BENEDITTI, T. R. B.; MAZO, G. Z. Aspectos da autoimagem e autoestima de idosos ativos. Motriz, Rio Claro, v. 15, n. 4, p. 788-796, out./dez. 2009.

NELSON, M. E. et al. Physical activity and public health in older adults: recommendation from the American College of Sports Medicine and the American Heart Association. Medicine Science Sports Exercice, v. 39, n. 8, p. 1435-45, August 2007. Doi: 10.1249/mss.0b013e3180616aa2.

OLIVEIRA, F. L. B.; ALMEIDA JÚNIOR, J. J. Extensão universitária: contribuições na formação de discentes de Enfermagem. Rev. Bras. Pesq. Saúde, Vitória, v. 17, n. 1, p. 19-24, jan./mar. 2015.2 Disponível em: http://www.portaldepublicacoes.ufes.br/RBPS/article/viewFile/12445/8655. Acesso em: 19 ago. 2019.

PILGER, C.; MENON, M. U.; MATHIAS, T. A. F. Utilização de serviços de saúde por idosos vivendo na comunidade. Rev. Esc. Enferm. USP, São Paulo, v. 47, n. 1, p. 213-220, 2013. Doi: 10.1590/S0080-62342013000100027.

SALIN, M. S. et al. Atividade física para idosos: diretrizes para implantação de programas e ações. Rev. Bras. Geriatr. Gerontol., Rio de Janeiro, v. 14, n. 2, p. 197-208, 2011. Doi: 10.1590/S1809-98232011000200002.

SANTOS, P. L.; FORONI, P. M.; CHAVES, M. C. F. Atividades físicas e de lazer e seu impacto sobre a cognição no envelhecimento. Medicina, Ribeirão Preto, v. 42, n. 1, p. 54-60, 2009. Doi: 10.11606/issn.2176-7262.v42i1p54-60.

SANTOS, R. L. Relato de experiência: importância da atividade física na qualidade de vida de idosas do "Grupo Renascer". 2014. Monografia (Graduação em Educação Física) - Centro de Ciências Biológicas e da Saúde, Universidade Estadual da Paraíba, Campina Grande, 2014.

SIQUEIRA, F. V. et al. Aconselhamento para a prática de atividade física como estratégia de educação à saúde. Cadernos de Saúde Pública, Rio de Janeiro, v. 25, n. 1, p. 203-213, 2009. Doi: 10.1590/S0102-311X2009000100022.

YOKOYAMA, C. E.; CARVALHO, R. S.; VIZZOTTO, M. M. Qualidade de vida na velhice segundo a percepção de idosos frequentadores de um centro de referência. Psicólogo inFormação, São Paulo, v. 10, n. 10, p. 57-82, jan./dez. 2006.

Submetido em 24 de setembro de 2019.

Aprovado em 30 de janeiro de 2020. 